\title{
Associations of alcohol use, mental health and socioeconomic status in England: \\ findings from a representative population survey
}

Jo-Anne Puddephatt ${ }^{\mathrm{a}^{*}}$, Andrew Jones ${ }^{\mathrm{a}}$, Suzanne H. Gage ${ }^{\mathrm{a}}$, Nicola T. Fear ${ }^{\mathrm{bc}}$, Matt Field ${ }^{\mathrm{d}}$, Sally McManus $^{\text {ef }}$, Orla McBride ${ }^{\mathrm{g}} \&$ Laura Goodwin ${ }^{\mathrm{a}}$

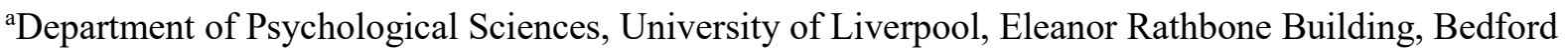
Street South, Liverpool, L69 7ZA, United Kingdom

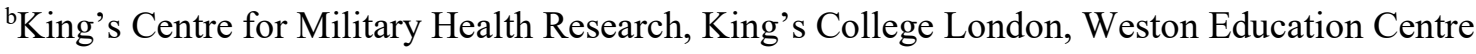
10 Cutcombe Road, London, SE5 9RJ, United Kingdom

'Academic Department of Military Mental Health, King's College London, Weston Education Centre 10 Cutcombe Road, London, SE5 9RJ, United Kingdom

${ }^{\text {d} D e p a r t m e n t ~ o f ~ P s y c h o l o g y, ~ U n i v e r s i t y ~ o f ~ S h e f f i e l d, ~} 1$ Vicar Ln, Sheffield City Centre, Sheffield, S1 2LT, United Kingdom

'National Centre for Social Research, 35 Northampton Square, London, EC1V 0AX, United Kingdom fSchool of Health Sciences, City University, Northampton Square, London, EC1V 0HB, United Kingdom

gSchool of Psychology, Ulster University, Cromore Road, Coleraine, Co. Londonderry, BT52 1SA, United Kingdom

i *Corresponding author: Jo-Anne Puddephatt, 2.80 Eleanor Rathbone Building, Bedford Street South, University of Liverpool, L69 7ZA. Email: joannep@liverpool.ac.uk 


\begin{abstract}
Background: Alcohol use and mental health problems often co-occur, however, little is known about how this varies by type of mental health problem and to what extent associations are explained by socioeconomic status (SES). Our study examined the prevalence and associations of non-drinking, hazardous use, and harmful/probable dependence in individuals who do and do not meet criteria for different mental health problems and whether associations remained after adjustment for SES.
\end{abstract}

Methods: A secondary analysis of an English dataset, 2014 Adult Psychiatric Morbidity Survey $(N=7,218)$, was conducted. The Alcohol Use Disorder Identification Test was used to categorise participants as non-drinking, low risk, hazardous use and harmful/probable dependence. Mental health problems were screened using a range of validated tools. Multinomial logistic regression analyses were used to address study aims.

Results: The prevalence of non-drinking, hazardous and harmful/probable dependence was higher among those meeting criteria for a mental health problem. After adjustment for SES, non-drinking was most common in those meeting criteria for probable psychotic disorder $(\mathrm{MOR}=3.42,95 \% \mathrm{CI}=1.74$ 6.70 ), hazardous use in those meeting criteria for anti-social personality disorder ( $\mathrm{MOR}=2.66$, $95 \% \mathrm{CI}=1.69-4.20)$ and harmful/probable dependence in those meeting criteria for borderline personality disorder $(\mathrm{MOR}=9.77,95 \% \mathrm{CI}=4.81-19.84)$.

Conclusions: There were marked increases in the odds of reporting both non-drinking and harmful drinking among those meeting criteria for a mental health problem, particularly more severe problems. Our findings indicate that the relationship between alcohol and mental health is more complex and comorbid alcohol and mental health problems should be treated in parallel with access to both services.

Keywords: alcohol use, mental health, comorbidity, socioeconomic characteristics, epidemiology

Word count: 4228 


\section{Introduction}

Almost a fifth of adults in England drink alcohol at hazardous or harmful levels (Drummond, 2016), and around one in six report symptoms of a common mental disorder (CMD) in the past week. The proportion of people reporting severe CMD symptoms has increased in recent years (Stansfeld et al., 2016). Individuals with a mental health problem, such as depression, are more likely to misuse alcohol (Bell \& Britton, 2014; Lai, Cleary, Sitharthan, \& Hunt, 2015). Longitudinal and prospective cohort research has found that individuals with depressed mood, psychosis or anxiety at baseline were at increased risk of reporting alcohol problems, dependence or alcohol use disorder at follow up (Crum et al., 2008; Degenhardt et al., 2019; Torvik et al., 2019) indicating that as mental health worsens alcohol use increases. While those with severe mental illnesses (SMI), such as bipolar disorder, are more likely to have a comorbid alcohol use disorder compared to those with a CMD (Grant et al., 2015; Hartz et al., 2014). Those with a mental health problem may also be more likely to not drink with evidence showing individuals with personality disorder being more likely to abstain than consume alcohol (Skogen et al., 2011) suggesting that alcohol and mental health may be more complex. Both heavy and non-drinkers have been found to have poorer physical health than low risk drinkers, known as the Jshaped curve (Gmel et al., 2003; White et al., 2002). However, this has been contested as providing evidence of the protective effects of moderate drinking because non-drinkers may include previous drinkers who have given up due to pre-existing health conditions (Day \& Rudd, 2019; Stockwell et al., 2016).

Alcohol may be used to cope with negative affect and reduce symptoms of poor mental health (Collins et al., 2018; Cooper et al., 1995; Holahan et al., 2002). Longitudinal research exploring associations of mental health and alcohol use found strongest evidence for a model in which declining mental health was most predictive of increasing alcohol use (Bell \& Britton, 2014) indicating that alcohol was used to self-medicate (Khantzian, 1997). There may be variations in how people with different mental health conditions use alcohol, for example someone with bipolar disorder may use alcohol differently from someone with post-traumatic stress disorder (Goodwin et al., 2017). A recent study found only social anxiety disorder was longitudinally associated with alcohol use disorder while other anxiety disorders were not indicating differences between specific types of mental health problems (Torvik et al., 2019). However, much of the research has examined associations of types of alcohol misuse or non-drinking, and restricted to specific types of mental health problems, separately (Crum et al., 2008; Degenhardt et al., 2018; Grant et al., 2015; Skogen et al., 2011; Torvik et al., 2019), therefore it is less well understood whether the pattern of alcohol misuse and non-drinking is similar across different types of mental health problems. 
Socioeconomic status (SES) is independently associated with both alcohol use and mental health problems, with those of lower SES more likely to experience alcohol harms and poor mental health than those of higher SES (Beard et al., 2019; Goodwin et al., 2017). Patterns of associations between SES and alcohol use depend on the assessment used; research has shown associations with binge-drinking and those on lower income and with A-Level education, whereas lower social grade and housing tenure was associated with decreased alcohol frequency and increased unit intake (Beard et al., 2019). The social causation hypothesis suggests that those experiencing more disadvantage, such as lower education, might disproportionately experience alcohol harms and poor mental health (Goldman, 1994). However, much of the research is limited to associations of SES with alcohol use or mental health separately (Beard, 2019; Goodwin et al., 2017), despite the availability of such data providing an opportunity to consider the role of SES with comorbid alcohol use and mental health problems.

Using an English dataset, the current study aimed to i) examine the prevalence and associations of non-drinking, hazardous and harmful/probable dependence in individuals who do and do not meet criteria for different mental health problems, ii) compare associations across different mental health problems, and iii) determine whether associations remained after adjustment for SES. We hypothesised that those meeting criteria for a mental health problem will be more likely to report non-drinking or harmful/probable dependence than those not meeting criteria and that these associations will be partially explained by demographic (e.g. age) and SES (e.g. education) characteristics. Finally, we hypothesised that non-drinking or harmful/probable dependence will be more strongly associated with more severe mental health problems.

\section{Method}

\subsection{Study design}

A secondary analysis of the 2014 Adult Psychiatric Morbidity Survey (APMS) was conducted with study plans pre-registered on Open Science Framework (https://osf.io/32xka/). The APMS uses a stratified, multi-stage random probability sample. It is a cross-sectional survey conducted among those living in private households in England every seven years since 1993. 2014 APMS data was accessed with special permission from NHS Digital (ref. DARS-NIC-220105-B3Z3S-v0.3).

\subsection{Participants and setting}

Further details of the methodology of APMS are described elsewhere (McManus et al., 2019). An advance letter introducing the survey was sent to each sampled address, one adult aged 16 or older was selected in each eligible household to take part in a face-to-face interview and reimbursed with a $£ 15$ high street voucher. Interviews were conducted in participants' homes, with some information collected by self-completion using computer assisted interviewing. $6 \%$ of participants did not complete the selfcompletion section of the interview. Interviews were conducted from May 2014 to September 2015 (McManus et al., 2019). 


\subsection{Measures}

Alcohol use: Past year alcohol use was measured using two screening questions and the Alcohol Use Disorder Identification Test (AUDIT); "Do you ever drink alcohol nowadays?", those who responded "no" were asked an additional question "Could I just check, does that mean you never have an alcoholic drink nowadays, or do you have an alcoholic drink very occasionally, perhaps for medicinal purposes or on special occasions like Christmas or New Year?". Those who responded "no" did not complete the AUDIT. The AUDIT is a 10-item questionnaire used to assess alcohol frequency, harmful use and consequences of drinking alcohol (Babor, Higgins-Biddle, Saunders, Monteiro, \& World Health Organization, 2001) with good internal reliability within this sample (Cronbach's $\alpha=0.96$ ). The following categories were used according to recommendations (Babor et al., 2001). Those with an AUDIT score of 0 or responding 'No' to screening questions were categorised as "non-drinkers". Those with an AUDIT score of 1-7 were categorised as "low risk" (reference category), 8-15 categorised as "hazardous use", 16 or more categorised as "harmful/probable dependence". Harmful use and probable dependence were combined due to small numbers.

Any CMD, depression, anxiety disorders, and phobia: The Clinical Interview Schedule-Revised (CIS$\mathrm{R})$ was used to screen for meeting criteria for a CMD. This was based on a participant's overall CIS-R score where a score of 12 to 17 categorised participants as having "moderate symptoms of CMD" and a score of 18 or more as having "severe symptoms of CMD". This was also used to screen for those meeting criteria for $10^{\text {th }}$ International Classification of Disease depression, anxiety and phobia diagnosis categories (Stansfeld et al., 2016). Due to small cell sizes, screening for mild, moderate and severe depression were grouped as "depression". Generalised anxiety disorder, obsessive compulsive disorder and panic disorder grouped as "anxiety". Social phobia, specific phobia and agoraphobia grouped as "phobia". Participants were also grouped as meeting criteria for any one (depression alone, anxiety alone or phobia alone), any two (two of depression, anxiety and/or phobia) or all three CMDs.

Probable PTSD: The PTSD Checklist (PCL-C) questionnaire is a self-report scale comprising 17-items which correspond to symptoms of DSM-IV PTSD in the past month. Participants were categorised as meeting criteria for probable PTSD if they had a score of 50 on the PCL-C and positive responses to at least one item on re-experiencing, three on avoidance and numbing, and two on hyperarousal (Fear, Bridges, Hatch, Hawkins, \& Wessely, 2016).

Bipolar disorder: The Mood Disorder Questionnaire is a 13-item questionnaire assessing lifetime experience of manic or hypomanic symptoms and, if these have been experienced at the same time, caused moderate to serious problems and has been shown to be reliable (Cronbach's $\alpha=0.97$ ). Those who scored seven or more were also asked; "Have several of these ever happened during the same period of time?" and "How much of a problem did any of these cause you - like being unable to work; having family, money or legal troubles; getting into arguments or fights?". Participants screened for 
bipolar disorder if they answered "yes" to the first additional question, "moderate" or "severe problem" to the second additional question, and had a score of seven or more (Marwaha, Sal, \& Bebbington, 2016).

Anti-Social Personality Disorder (ASPD) and Borderline Personality Disorder (BPD): The Structured Clinical Interview for DSM-IV Personality Disorders was used to screen for lifetime ASPD and BPD (Moran, 2016). Meeting criteria for ASPD was defined as having a score of three or more on questions around failure to conform to social norms and aggressiveness, as well as meeting diagnostic criteria for conduct disorder before age 15. Meeting criteria for BPD was defined as having a score of five or more to questions related to instability of interpersonal relationships, mood, with impulsivity in childhood (Moran, 2016).

Probable psychotic disorder: The Psychosis Screening Questionnaire (PSQ) is a five-item questionnaire assessing symptoms of psychosis. Screening for probable psychotic disorder included participants who positively endorsed any two of the following and did not complete a follow-up interview: 1) currently taking antipsychotic medication; 2) reporting an inpatient stay for a mental or emotional problem in the past three months; or 3) having been admitted to a hospital specialising in mental health problems at any time, 5) a positive response to question 5a on the PSQ assessing auditory hallucinations, 6) reporting symptoms suggestive of psychotic disorder or 7) discussing such symptoms with a GP in the past year, self-reported identification with psychotic disorder (Bebbington, 2016).

Attention Deficit Hyperactivity Disorder (ADHD): The Adult ADHD Self-Report Scale-v1.1 is a sixitem shortened version of the 18-item Symptom Checklist scale which measures the frequency of recent symptoms of adult ADHD and is shown to be reliable (Cronbach's $\alpha=0.73$ ). A score of four or more was used to screen positive for ADHD (Brugha, 2016).

Demographic and SES characteristics: A range of demographic and SES characteristics were measured. Gender ("male" as the reference category), age (categorised as "16-34", "35-54" (reference category), "55-74", "75+"), marital status (categorised as "married or civil partnership" (reference category), "single", "separated/divorced/widowed"), ethnicity (categorised as "white" (reference category) and "non-white", this variable was not pre-registered), whether participants had children aged under 16 years living in the household (categorised as "yes" or "no" (reference category), education (categorised as "degree level or above", "GCSE or A-Level" (reference category), "foreign qualifications", "no qualifications"), occupational grade (categorised as "professional/managerial", "intermediate, lower supervisory/small employers and own account workers", "lower supervisory technical/semi-routine/routine" (reference category), "never worked/not worked in past year" and "not classified for other reason", housing tenure (categorised as "owner-occupied" (reference category), "private renter", and "social or other renter") (McManus, Bebbington, Jenkins, \& Brugha, 2016).

\subsection{Sample size}


Of the 13,313 addresses identified in the sampling frame the final 2014 APMS sample was 7,546, with a response rate of $57 \%$. This included 18 partial interviews where the participant completed some sections of the full interview. Most variables had a small amount of missing data $(\sim 0.01 \%)$ though alcohol use, probable PTSD and bipolar disorder had 3.7\%, 5.3\% and 5.5\% missing data, respectively. Given the small amount of missing data, a complete case analysis was conducted with participants who completed the alcohol use measures $(N=7,218)$ as this was our main outcome variable. Potential nonresponse bias could be due to items being in the self-completion section of the questionnaire (McManus, et al., 2019).

\subsection{Statistical methods}

Cross-tabulations were used to examine the prevalence of the different alcohol use categories in individuals who did and did not meet criteria for each mental health problem. The unweighted frequency and weighted percentage were reported, with weights accounting for selection probabilities and nonresponse. Alcohol use categories (non-drinking, hazardous use, harmful/probable dependence) were our outcome variables and meeting criteria for CMD symptoms and specific mental health problems predictor variables.

Unadjusted multinomial logistic regression was conducted to examine the strength of the association between non-drinking, hazardous use, and harmful/probable dependence and meeting criteria for symptoms of CMD and specific mental health problems compared to those who did not meet criteria for symptoms of CMD and the respective mental health problem, respectively. We then adjusted our analyses for demographic (gender, age, marital status, ethnicity and having children in the household) and SES characteristics (occupational grade, education and housing tenure). Multinomial odds ratios and $95 \%$ confidence intervals were used to assess and compare the size of associations between alcohol use and specific mental health problems. Confidence intervals overlapping with 1 and with a p-value of over 0.05 were deemed non-significant. Forest plots were produced to illustrate the strength of the association between alcohol use categories and mental health problems. All analyses were conducted in STATA 14.0 using the 'svy' command.

A pre-registered sensitivity analysis was conducted to examine the interaction between gender and CMD symptoms on alcohol use outcomes (non-drinking, hazardous use, and harmful/probable dependence). We conducted an exploratory analysis to examine the prevalence and associations between meeting criteria for one, two or all CMD, or any SMI with alcohol use categories.

\section{Results}

\subsection{Descriptive data}

Table 1 shows participant demographic and SES characteristics, and associations with alcohol use. Nondrinkers were more likely to be female, aged under 35 or over 74, non-white, previously partnered, had 
children aged under 16 living in the household, no qualifications, not worked in the last year and living in rented accommodation. Hazardous or harmful/probable dependent drinkers were more likely to be male and single. Being of higher occupational grade or not working in the last year decreased the likelihood of harmful/probable dependent drinking.

\subsection{Primary analyses}

\subsubsection{Non-drinking and meeting criteria for mental health problems}

The prevalence of non-drinking was higher among those with moderate $(22.72 \%)$ and severe $(33.21 \%)$ symptoms of CMD compared to those without symptoms (21.81\%, see table 2$)$. The prevalence of nondrinking among those meeting criteria for a specific mental health problem was highest for probable psychotic disorder (52.30\%, see table 2). With the exception of ASPD, the prevalence of non-drinking was higher among those meeting criteria for any specific mental health problem compared to those not meeting criteria for the respective problem (see table 2).

Those meeting criteria for severe symptoms of CMD were twice as likely to report non-drinking and associations remained, though slightly attenuated, after adjustment for demographic and SES characteristics (see table 3). Adjusted associations with non-drinking increased for most mental health problems with odds highest for more severe problems, such as probable psychotic disorder, whereby odds were three-fold indicating a moderate effect. Unadjusted associations between meeting criteria for specific mental health problems and non-drinking (excluding ASPD) were partially attenuated after adjustment for demographic and SES characteristics, excluding anxiety disorder and ADHD where associations were no longer significant (see table 3). This suggests that non-drinking is associated with most mental health problems after accounting for participant SES characteristics and strongest for those meeting criteria for more severe problems (see figures 1 and 2 for further illustration).

\subsubsection{Hazardous use and meeting criteria for mental health problems}

There was no association between hazardous use and having symptoms of CMD (see table 3). For specific mental health problems, the prevalence of hazardous use was highest for those meeting criteria for ASPD (30.29\%, see table 2) with a two and a half fold increase in the odds of reporting hazardous use after adjustment for demographic and SES characteristics, compared to low risk, indicating a moderate effect (see table 3). There were some associations between meeting criteria for mental health problems and hazardous use, excluding depression, phobia and probable psychotic disorder, which were partially attenuated after adjustment for demographic and SES characteristics (see table 3). Associations were strongest for personality disorder and bipolar disorder whereby odds were over seven-fold indicating a large effect, suggesting that those meeting criteria for more severe problems were more likely to report hazardous drinking, compared with low risk and this is reflected in the forest plots (see figures 1 and 2). 


\subsubsection{Harmful/probable dependence and meeting criteria for mental health problems}

The prevalence of harmful/probable dependence among those with moderate $(6.47 \%)$ or severe symptoms $(8.54 \%)$ of CMD was higher compared to those without symptoms $(2.27 \%$, see table 2$)$. The prevalence of harmful/probable dependence was higher among those meeting criteria for all mental health problems (see table 2).

Moderate to large effects were found with three- and five-fold increases in the odds of harmful/probable dependence for those with moderate or severe CMD symptoms, compared to low risk, respectively after adjustment for demographic and SES characteristics (see table 3). For more specific mental health problems, associations were strongest for those meeting criteria for more severe problems; individuals with BPD had an almost 10-fold increase in the odds, compared to low risk, indicating a large effect (see table 3). Associations with specific mental health problems, excluding probable psychotic disorder, remained after adjustment for demographic and SES characteristics (see table 3 ) and this is reflected in the forest plots (see figures 1 and 2). The attenuated effect for meeting criteria for probable psychotic disorder may be due to insufficient power (see table 3).

\subsection{Exploratory analyses}

The prevalence and associations of non-drinking or harmful/probable dependence was higher among those meeting criteria for one or more CMDs or SMIs compared to those not meeting criteria, with moderate effect sizes (see supplementary tables 1 and 2).

\subsubsection{Sensitivity analyses}

A sensitivity analysis found no significant interaction between gender and CMD symptoms for any of the alcohol use outcomes ( $>0.05$, see supplementary table 3 ).

\section{Discussion}

\subsection{Key findings}

Supporting our hypotheses, we found those meeting criteria for a mental health problem were more likely to report non-drinking, hazardous and harmful/probable dependence, compared to low risk. Associations were strongest for those meeting criteria for probable psychotic disorder, ASPD and BPD, respectively, even after adjustment for demographic and SES characteristics. Our study shows that whilst alcohol misuse is associated with mental health problems, which is consistent with previous research, we also found non-drinking is strongly associated with such problems, even after accounting for demographic and SES characteristics. This is one of the first studies to examine associations between alcohol use and non-drinking across a range of mental health problems while also examining the role of SES in explaining comorbidity. 
Our findings indicate the need to explore additional mechanisms of alcohol use and nondrinking among those with a mental health problem, specifically those meeting criteria for more severe problems. Further, our findings strengthen the notion that alcohol use and mental health problems should be treated in parallel, with improved communication across mental health and substance use services.

\subsection{Comparisons to previous research}

Our findings are partially consistent with previous research which found that alcohol use was higher among those with a mental health problem (Bell \& Britton, 2014; Swendsen et al., 2010), specifically depressive, anxiety and more severe mental health problems (Grant et al., 2015). A recent study found the prevalence of hazardous/harmful alcohol use was highest for those with depression compared to other disorders whereas we found this was highest for those with more severe problems, but this may be due to differences between the cut-offs used (Davis et al., 2020). However, much of the research has focussed on alcohol misuse and non-drinking separately, and with regards to a restricted number of mental health problems (Crum et al., 2008; Degenhardt et al., 2018; Skogen et al., 2011). We have shown, in a sample representative of the general population, moderate to strong associations between alcohol misuse and non-drinking across different mental health problems, particularly those meeting criteria for a SMI, such as probable psychotic disorder.

Strong associations were found between non-drinking and mental health problems, particularly more severe problems, indicating that alcohol use and mental health comorbidity may extend beyond alcohol misuse. Specifically, only BPD, and not ASPD, was associated with non-drinking, which is in contrast to a previous study (Skogen et al., 2011), indicating that non-drinking may be uniquely associated with specific types of personality disorder. Reasons for non-drinking may include participants' beliefs, severity of the illness, being on medication, or being a previous harmful drinker (Healey et al., 2009; Skogen et al., 2011). Indeed, longitudinal research has shown that drinkers at baseline who stopped drinking at follow-up were more likely to report an onset of depression or anxiety, therefore, worsened mental health may influence the decision to not drink (Sarich et al., 2019). Our findings of non-drinking indicate having a mental health problem may not necessarily increase the likelihood of drinking at harmful levels and other factors may influence drinking or abstinence among those with a mental health problem. Currently, there is limited research exploring the relationship between non-drinking and mental health, and our findings suggest a need to examine this further.

We found those meeting criteria for anxiety, BPD or ADHD were more likely to drink alcohol at hazardous or harmful/probable dependent levels with stronger associations for harmful/probable dependence which is consistent with previous research (Grant et al., 2015; Lai et al., 2015). It may be that alcohol is used to control different phases of an illness, for example, used to control the feelings of elation in the lead up to the manic phase and used less in the depression phase (Healey et al., 2009; Lai et al., 2015). Longitudinal research has shown that those reporting poor mental health, psychotic experiences or depressive mood at baseline were at an increased risk of reporting alcohol problems 
(Bell \& Britton, 2014; Degenhardt et al., 2018; Torvik et al., 2017) indicating that alcohol may be used to cope worsened mental health and our findings support this.

Stronger associations with alcohol use were found among those meeting criteria for more severe problems which could be explained by theories, such as the self-medication theory (Khantzian, 1997). Indeed, it may be that experiencing specific symptoms associated with more severe mental health problems increases the likelihood of drinking (Healey et al., 2009; Tragesser et al., 2007), this could be prevented by the treatment of symptoms (Swendsen, 2010). It was not possible to explore whether alcohol use was motivated by mental health symptoms in this study, however, some theories suggest that alcohol can be used to cope with poor mental health (Cooper et al., 1995), and research has shown this for some mental health problems, such as depression and anxiety (Collins et al., 2018; Holahan et al., 2002). Our findings suggest a need to explore this across a range of more severe problems which might inform how to tailor interventions more appropriately.

Finally, we found that demographic and SES characteristics only partially accounted for the association between alcohol use and mental health problems suggesting that, although such characteristics should be considered, alcohol use remains strongly associated with mental health problems irrespective of an individual's SES characteristics. More specifically, those with a mental health problem were more likely to drink harmfully and this was not restricted to those of lower SES which is contrary to previous research (Beard et al., 2019) and theories such as the social causation hypothesis (Goldman, 1994). Our findings, instead, suggest that alcohol may be used as a coping technique for mental health across SES groups. Associations may differ depending on which SES measure is used, and that methods such as latent class analysis can be used to define more descriptive categories beyond just high or low SES (Skogen, Boe, Thorrison, Riper \& Aas, 2019).

\subsection{Strengths and limitations}

This study examined the prevalence and association between a large range of mental health problems and alcohol use in England. Much of the previous research has focused on alcohol misuse or nondrinking separately (Grant et al., 2015; Skogen et al., 2011; Swendsen et al., 2010) and research that has focussed on investigating the associations with mental health problems, has restricted this to a small number of problems (Goodwin et al., 2017; Skogen et al., 2011; Thandi et al., 2015). Our study provides a novel insight into the associations between alcohol and mental health while considering other characteristics.

Our study has limitations. First, the APMS is a cross-sectional survey, and therefore, we cannot determine the timeline of associations between mental health and alcohol use. Second, while measures used in our study have been validated, these are screening tools and those meeting criteria for a specific mental health problem would require further investigation to determine diagnostic caseness. Third, where possible we have used stringent criteria to screen for mental health problems though some use diagnostic criteria (i.e. PCL-C) and others do not (i.e. mood disorder questionnaire), and comparing different problems is not always directly comparable. And fourth, while we included a range of 
demographic and SES characteristics in our analyses, other characteristics such as income may explain some of these associations, however, it was not possible to do this in the current study due to a large amount of missing data.

\subsection{Conclusions}

Our study found meeting criteria for mental health problems, in particular SMIs, was strongly associated with drinking above hazardous levels and non-drinking which remained after adjustment for demographic and SES characteristics using a large representative dataset of England. Our findings suggest that treatment for alcohol use and mental health should be addressed in parallel and comorbidity is not restricted to individuals of lower SES. Further, mechanisms underlying misusing alcohol and non-drinking among those with more severe mental health problems should be explored.

\section{References}

Babor, T. F., Higgins-Biddle, J. C., Saunders, J. B., Monteiro, M. G., \& World Health Organization. (2001). AUDIT: the alcohol use disorders identification test: guidelines for use in primary health care.

Beard, E., Brown, J., West, R., Kaner, E., Meier, P., \& Michie, S. (2019). Associations between socioeconomic factors and alcohol consumption: A population survey of adults in England. PLOS ONE, 14(2), e0209442. doi:10.1371/journal.pone.0209442

Bebbington, P., Rai D., Strydom A., Brigha T., McManus S. \& Morgan Z. (2016). Chapter 5: Psychotic disorder. In Mental health and wellbeing in England: Adult Psychiatric Morbidity Survey 2014. Leeds: NHS Digital.

Bell, S., \& Britton, A. (2014). An exploration of the dynamic longitudinal relationship between mental health and alcohol consumption: a prospective cohort study. BMC medicine, 12(1), 91.

Brugha T., A. P., Strydom A., Morgan Z. \& Christie S. (2016). Chapter 8: Attentiondeficit/hyperactivity disorder. In Mental health and wellbeing in England: Adult Psychiatric Morbidity Survey 2014. Leeds: NHS Digital.

Collins, J. L., Thompson, K., Sherry, S. B., Glowacka, M., \& Stewart, S. H. (2018). Drinking to cope with depression mediates the relationship between social avoidance and alcohol problems: A 3-wave, 18-month longitudinal study. Addictive behaviors, 76, 182-187.

Cooper, M. L., Frone, M. R., Russell, M., \& Mudar, P. (1995). Drinking to regulate positive and negative emotions: a motivational model of alcohol use. Journal of Personality and Social Psychology, 69(5), 990.

Crum, R. M., Green, K. M., Storr, C. L., Chan, Y. F., Ialongo, N., Stuart, E. A., \& Anthony, J. C. (2008). Depressed mood in childhood and subsequent alcohol use through adolescence and young adulthood. Archives of general psychiatry, 65(6), 702-712.

Davis, K. A., Coleman, J. R., Adams, M., Allen, N., Breen, G., Cullen, B., ... \& Howard, L. M. (2020). Mental health in UK Biobank-development, implementation and results from an online questionnaire completed by 157366 participants: a reanalysis. British Journal of Psychiatry Open, 6(2).

Day, E., \& Rudd, J. H. (2019). Alcohol use disorders and the heart. Addiction, 114(9), 1670-1678.

Degenhardt, L., Saha, S., Lim, C. C., Aguilar-Gaxiola, S., Al-Hamzawi, A., Alonso, J., ... \& de Girolamo, G. (2018). The associations between psychotic experiences and substance use and substance use disorders: findings from the World Health Organization World Mental Health surveys. Addiction, 113(5), 924-934.

Drummond, C., McBride, O., Fear, N., Fuller, E. (2016). 'Chapter 10: Alcohol dependence' in McManus S, Bebbington P, Jenkins R, Brugha T. (eds) Mental health and wellbeing in England: Adult Psychiatric Morbidity Survey 2014. Leeds: NHS Digital.

Fear, N. T., Bridges, S., Hatch, S., Hawkins, V., \& Wessely, S. (2016). Chapter 4: Posttraumatic stress disorder. In Mental health and wellbeing in England: Adult Psychiatric Morbidity Survey 2014. Leeds: NHS Digital. 
Gmel, G., Gutjahr, E., \& Rehm, J. (2003). How stable is the risk curve between alcohol and all-cause mortality and what factors influence the shape? A precision-weighted hierarchical metaanalysis. European journal of epidemiology, 18(7), 631-642.

Goldman, N. (1994). Social factors and health: the causation-selection issue revisited. Proceedings of the National Academy of Sciences, 91(4), 1251-1255.

Goodwin, L., Gazard, B., Aschan, L., MacCrimmon, S., Hotopf, M., \& Hatch, S. (2017). Taking an intersectional approach to define latent classes of socioeconomic status, ethnicity and migration status for psychiatric epidemiological research. Epidemiology and psychiatric sciences, 1-12.

Goodwin, L., Norton, S., Fear, N. T., Jones, M., Hull, L., Wessely, S., \& Rona, R. J. (2017). Trajectories of alcohol use in the UK military and associations with mental health. Addictive Behaviors, 75 , 130-137. doi:https://doi.org/10.1016/j.addbeh.2017.07.010

Grant, B. F., Goldstein, R. B., Saha, T. D., Chou, S. P., Jung, J., Zhang, H., . . Huang, B. (2015). Epidemiology of DSM-5 alcohol use disorder: results from the National Epidemiologic Survey on Alcohol and Related Conditions III. JAMA psychiatry, 72(8), 757-766.

Hartz, S. M., Pato, C. N., Medeiros, H., Cavazos-Rehg, P., Sobell, J. L., Knowles, J. A., . . Genomic Psychiat Cohort, C. (2014). Comorbidity of Severe Psychotic Disorders With Measures of Substance Use. JAMA psychiatry, 71(3), 248-254. doi:10.1001/jamapsychiatry.2013.3726

Healey, C., Peters, S., Kinderman, P., McCracken, C., \& Morriss, R. (2009). Reasons for substance use in dual diagnosis bipolar disorder and substance use disorders: A qualitative study. Journal of affective disorders, 113(1-2), 118-126.

Holahan, C. J., Moos, R. H., Holahan, C. K., Cronkite, R. C., \& Randall, P. K. (2003). Drinking to cope and alcohol use and abuse in unipolar depression: a 10-year model. Journal of abnormal psychology, 112(1), 159.

Khantzian, E. J. (1997). The self-medication hypothesis of substance use disorders: a reconsideration and recent applications. Harvard review of psychiatry, 4(5), 231-244.

Lai, H. M. X., Cleary, M., Sitharthan, T., \& Hunt, G. E. (2015). Prevalence of comorbid substance use, anxiety and mood disorders in epidemiological surveys, 1990-2014: A systematic review and meta-analysis. Drug and Alcohol Dependence, 154, 1-13. doi:https://doi.org/10.1016/j.drugalcdep.2015.05.031

Marwaha, S., Sal, N., \& Bebbington, P. (2016). Chapter 9: Bipolar disorder. In Mental health and wellbeing in England: Adult Psychiatric Morbidity Survey 2014. Leeds: NHS Digital.

McManus, S., Bebbington, P., Jenkins, R., \& Brugha, T. (2016). Mental Health and Wellbeing in England: Adult Psychiatric Morbidity Survey 2014: a Survey Carried Out for NHS Digital by NatCen Social Research and the Department of Health Sciences, University of Leicester: NHS Digital.

McManus, S., Bebbington, P. E., Jenkins, R., Morgan, Z., Brown, L., Collinson, D., \& Brugha, T. (2019). Data resource profile: Adult Psychiatric Morbidity Survey (APMS). International journal of epidemiology. doi:10.1093/ije/dyz224

McManus, S., Gunnell, D., Cooper, C., Bebbington, P. E., Howard, L. M., Brugha, T., . . Appleby, L. (2019). Prevalence of non-suicidal self-harm and service contact in England, 2000-14: repeated cross-sectional surveys of the general population. The Lancet Psychiatry, 6(7), 573-581.

Moran, P., Rooney, K., Tyrer P., Coid J. . (2016). Chapter 7: Personality disorder. In Mental health and wellbeing in England: Adult Psychiatric Morbidity Survey 2014. Leeds: NHS Digital.

Sarich, P., Canfell, K., Banks, E., Paige, E., Egger, S., Joshy, G., ... \& Weber, M. (2019). A prospective study of health conditions related to alcohol consumption cessation among 97,852 drinkers aged 45 and over in Australia. Alcoholism: Clinical and Experimental Research, 43(4), 710721.

Skogen, J. C., Bøe, T., Thørrisen, M. M., Riper, H., \& Aas, R. W. (2019). Sociodemographic characteristics associated with alcohol consumption and alcohol-related consequences, a latent class analysis of The Norwegian WIRUS screening study. BMC public health, 19(1), 1364. doi:10.1186/s12889-019-7648-6

Skogen, J. C., Mykletun, A., Ferri, C., Bebbington, P., Brugha, T., Coid, J., . . . Stewart, R. (2011). Mental and personality disorders and abstinence from alcohol: results from a national household survey. Psychological medicine, 41(4), 809-818. 
Stansfeld, S., Clark, C., Bebbington, P., King, M., Jenkins, R., \& Hinchliffe, S. (2016). Common mental disorders: Adult Psychiatric Morbidity Survey 2014. NHS Digital.

Stockwell, T., Zhao, J., Panwar, S., Roemer, A., Naimi, T., \& Chikritzhs, T. (2016). Do "moderate" drinkers have reduced mortality risk? A systematic review and meta-analysis of alcohol consumption and all-cause mortality. Journal of studies on alcohol and drugs, 77(2), 185-198.

Swendsen, J. (2010). Psychiatric and Substance Use Disorder Comorbidity.

Swendsen, J., Conway Kevin, P., Degenhardt, L., Glantz, M., Jin, R., Merikangas, K. R., . . Kessler, R. C. (2010). Mental disorders as risk factors for substance use, abuse and dependence: results from the 10-year follow-up of the National Comorbidity Survey. Addiction, 105(6), 1117-1128.

Thandi, G., Sundin, J., Ng-Knight, T., Jones, M., Hull, L., Jones, N., . . Fear, N. T. (2015). Alcohol misuse in the United Kingdom Armed Forces: A longitudinal study. Drug \& Alcohol Dependence, 156, 78-83.

Torvik, F. A., Rosenström, T. H., Gustavson, K., Ystrom, E., Kendler, K. S., Bramness, J. G., ... \& Reichborn-Kjennerud, T. (2019). Explaining the association between anxiety disorders and alcohol use disorder: A twin study. Depression and anxiety, 36(6), 522-532.

Tragesser, S. L., Sher, K. J., Trull, T. J., \& Park, A. (2007). Personality disorder symptoms, drinking motives, and alcohol use and consequences: cross-sectional and prospective mediation. Experimental and Clinical Psychopharmacology, 15(3), 282.

White, I. R., Altmann, D. R., \& Nanchahal, K. (2002). Alcohol consumption and mortality: modelling risks for men and women at different ages. British Medical Journal, 325(7357), 191.

\section{Funding}

This research was funded as part of a PhD Studentship by the Society for the Study of Addiction. 